\title{
Damage Detection for Civil Structural Health Monitoring Application - A Case Study of the Steel Grid Bridge Structural Model
}

\author{
Zoran MIŠKOVIĆ, Saad AL-WAZNI, Ahmed ALALIKHAN
}

\begin{abstract}
The procedure for detecting the location and severity of damage of complex structural systems using their modal properties is an important tool of Structural Health Monitoring (SHM) of civil infrastructure. The herein presented research proposes procedures for damage detection based on two heuristic optimization methods: Simulated Annealing (SA) and Tabu Search (TS). In order to test the proposed procedures in different frequency ranges, experimental and numerical analyses were conducted on a steel grid bridge model in two configurations, according to the total mass of the structure, as well as for two simulated damage cases. The calibration of model parameters, according to experimentally extracted modal properties, is carried out using the proposed procedures. Numerical computations were conducted using ANSYS package and developed routines under MATLAB environment for model calibration and damage detection procedures. Experimental modal properties were extracted from ambient vibration measurements, as state-of- the art in SHM of complex structures, by the Frequency Domain Decomposition (FDD) technique, using ARTeMIS software. Both of the proposed procedures for model calibration and damage detection, with adopted objective functions including frequency and mode shape differences, exhibit accuracy, efficiency and robustness.
\end{abstract}

Keywords: ambient vibrations; modal properties; model calibration; optimization methods; structural health monitoring

\section{INTRODUCTION}

Presence of damage in the structure may reduce its performance by decreasing the service life or even progress to cause a catastrophic failure [1]. The techniques that monitor the integrity of the structures are so-called Structural Health Monitoring-(SHM) procedures. The SHM is the art of extracting information on the existence, location and extent of damage in the structure using nondestructive methods [2], such as ambient vibration measurements.

The damage detection procedure in SHM applications includes the initial FE model calibration tuning of physical model parameters and modification of the calibrated FE model through artificially simulating position and damage severity of an element. Physical parameters include geometry and material properties, boundary conditions and additional mass [3] which have significant effect on modal properties. Various damage detection procedures could be found in the literature such as: change in natural frequencies; change in mode shapes, change in the structure flexibility matrix; change in the dynamic stiffness matrix and change in flexural stiffness of the structure [4]. In many damage detection procedures, optimization technique has to be adopted as the main part with adequate sensitive objective function to reflect changes of the included assumed parameters.

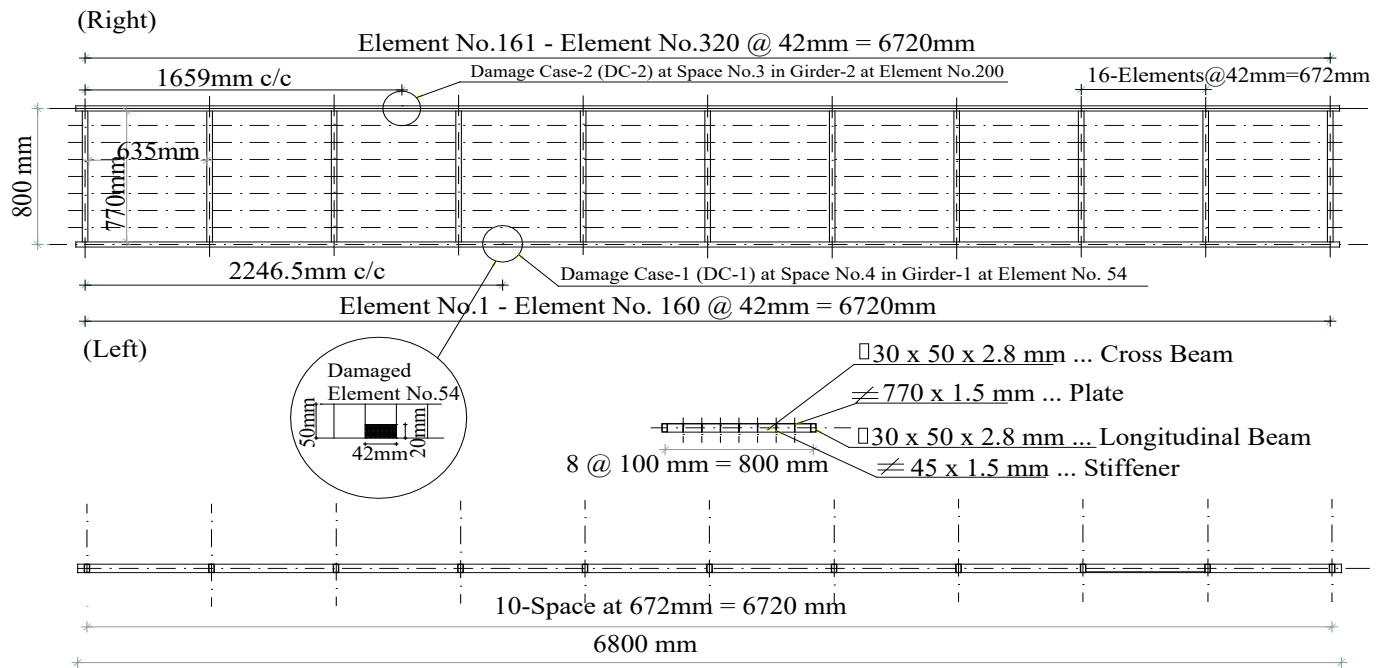

Figure 1 Layout of steel grid bridge structural model

Generally, the objective function consists of differences between the included target parameters, such as modal frequencies, mode shape vectors, MAC (Modal Assurance Criterion) values and strain energy residuals [5]. The optimization techniques are used to minimize such differences with respect to the variables included in the selected search space [6]. The search (design) space may be so large, with more local minima, and the applied optimization technique has to reach the global optimum or near optimal solution within a reasonable time frame. Classical techniques for the so-called hard nonlinear optimization problems are usually not satisfactory efficient or computationally expensive for solving such problems with more local minima [7]. Due to the ability of Simulated Annealing (SA) and Tabu Search (TS) to find global optimum or near-global optimum solution, to avoid local 
minima in the search space and their quick convergence performances $[8,9]$, heuristic optimization techniques are suitable for such complex and hard problems.

The present study proposes the application of two procedures, the first one includes only natural frequencies for model calibrations and the other one includes both mode frequencies and mode shapes for damage detection, using SA and TS heuristic optimization techniques. For damage detection procedure, changes in modal properties due to reduction of flexural stiffness have been adopted. Based on the sensitivity of modal properties, two different forms of objective function for the application of SA and TS optimization techniques were implemented during the testing phase $[10,11]$.

\section{THE ADOPTED GRID BRIDGE STRUCTURAL MODEL}

This study adopts a simply supported steel grid bridge model as a case study for damage detection. The model is $6800 \mathrm{~mm}$ long and $800 \mathrm{~mm} \mathrm{c} / \mathrm{c}$ wide with 10 grids length of $672 \mathrm{~mm}$. The main longitudinal edge and cross steel beams are rectangular hollow section dimensions of $30 \times$ $50 \times 2,8 \mathrm{~mm}$. The deck of the bridge model is formed as a thin orthotropic plate made of steel, with thickness of 1,5 $\mathrm{mm}$ and with 7 longitudinal stiffeners spaced on $100 \mathrm{~mm}$, Fig. 1.

All parts of the model are welded along the whole contact areas, Fig. 1. The initial values of material properties are assumed as follows: modulus of elasticity of $E$ initial $=200 \mathrm{GPa}$, Poisson's ratio $v=0,3$ and initial mass density $\rho$ initial $=7,860 \times 10^{3} \mathrm{~kg} / \mathrm{m}^{3}$. In order to get modal frequencies in different frequency ranges, the model was adopted for two cases: without additional mass and with additional mass, Fig. 2 and Fig. 3.

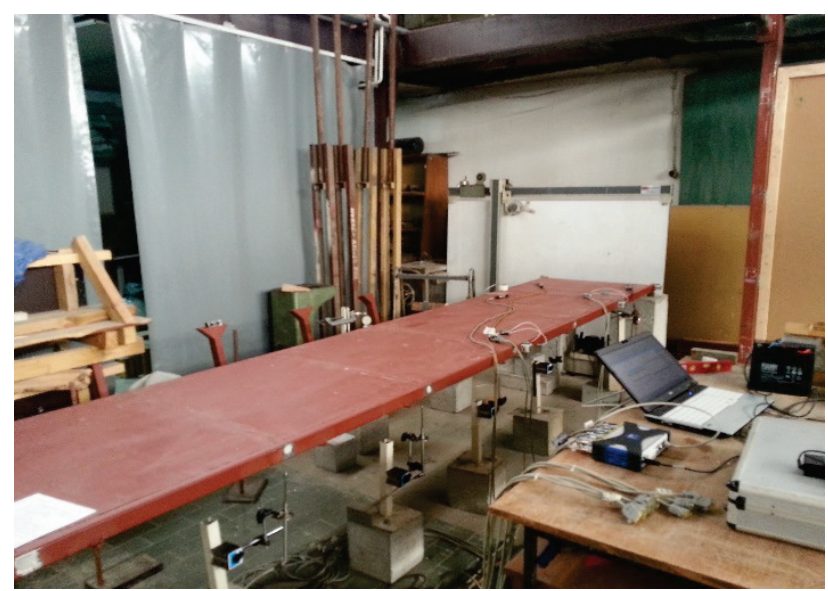

Figure 2 Grid bridge model during the test without additional mass

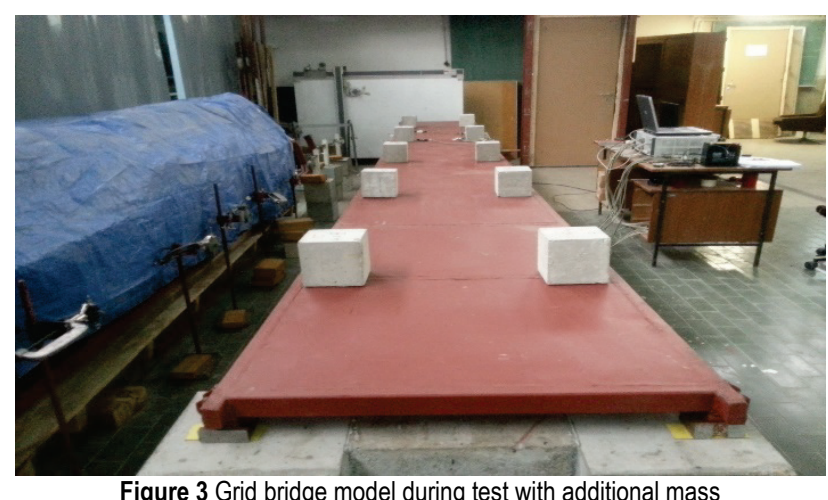

The case with additional mass included 10 masses (concrete cubes) placed at joints of the main longitudinal and cross beams inside the span, masses of $7,925 \mathrm{~kg}$, as shown in Fig. 3. In both cases, the model was supported at four points, with two hinges on one side and two roller supports on the other side in longitudinal direction.

\section{EXPERIMENTAL AND NUMERICAL ANALYSIS OF THE MODEL IN INTACT}

In order to extract real structural model vibration properties, the experimental modal test of the grid bridge model is carried out under simulated ambient vibrations at the Laboratory for Structures at the Faculty of Civil Engineering, University of Belgrade.

\subsection{Experimental Modal Analysis of the Model in Intact Case under Simulated Ambient Excitation}

Modal test is carried out using eight accelerometers, placed on the cubes glued on the intersecting nodes of the main and cross beams, as shown in Fig. 2. In order to get an adequate response for estimation procedure, it was necessary to record five sets of acceleration-time histories in vertical and lateral directions at each of the crossing nodes, Fig. 1. Ambient vibration simulation was carried out using a bass shaker, a music device which produces low vibrations in the range $5-200 \mathrm{~Hz}$, placed close to one support of the structure, Fig. 2. Low noise, high sensitivity and low frequency accelerometers from Silicon Designs Model 2400, with the range of DC-400 Hz, were used for acceleration measurements. Data acquisition was conducted by 24-bits 8 channel HBM-Hottinger Baldwin Messtechnik, QuantumX measuring amplifier, at the sampling frequency of $600 \mathrm{~Hz}$. Modal frequencies and mode shapes are extracted using ARTeMIS - extractor software, as the state-of- the-art software for the ambient vibration analysis. Out of few implemented extraction procedures, frequency domain decomposition (FDD) technique is adopted. Several trials of measurements were done to get more confidence of the extracted modal properties, according to the spectral density matrices estimated based on the recorded ambient vibration data. In all cases, the structural modes are estimated using peakpicking - according to FDD estimation procedure, as shown in Fig. 4. Tab. 1 summarizes the extracted modal properties of the first five modes by the ARTeMIS extractor of the recorded ambient vibration of the bridge model without additional mass.

\subsection{Numerical Modelling of the Model in Intact Case}

The FE beam model of the steel grid bridge structural model, Fig. 1, consists of 609 nodes and 928 elements with three element lengths (42 mm, $100 \mathrm{~mm}$ and $168 \mathrm{~mm})$. According to the importance of the main longitudinal beams for structural integrity, those parts are modelled with the smallest length of $42 \mathrm{~mm}$, to provide the possibility for determining the precise damage location. The length of beam elements of $100 \mathrm{~mm}$ is considered for the hidden cross beams in lateral direction of the orthotropic plate. Those elements are adopted to suppress local behaviour of longitudinal beams of the orthotropic 
plate. Thus, zero mass density is assumed for those elements with rectangular solid cross section dimensions of $(1,5 \times 100) \mathrm{mm}$. The length of $168 \mathrm{~mm}$ is used for elements of longitudinal stiffener beams of the orthotropic plate. The selection of different element lengths is adopted in order to reduce the number of elements of FE model. The study focused on 320 elements of longitudinal main girders, length of $42 \mathrm{~mm}$.

The numerical analysis is carried out using ANSYS package for structural analysis, Beam4 element type and linear elastic isotropic material. By means of the modal analysis, the first five modes of the intact case are computed for the assumed values of initial material properties used in FE model. The computed and experimentally estimated modal frequencies for the case without additional mass are listed in Tab. 1, with the corresponding differences. The corresponding experimentally and numerically computed mode shapes are presented in Fig. 5.

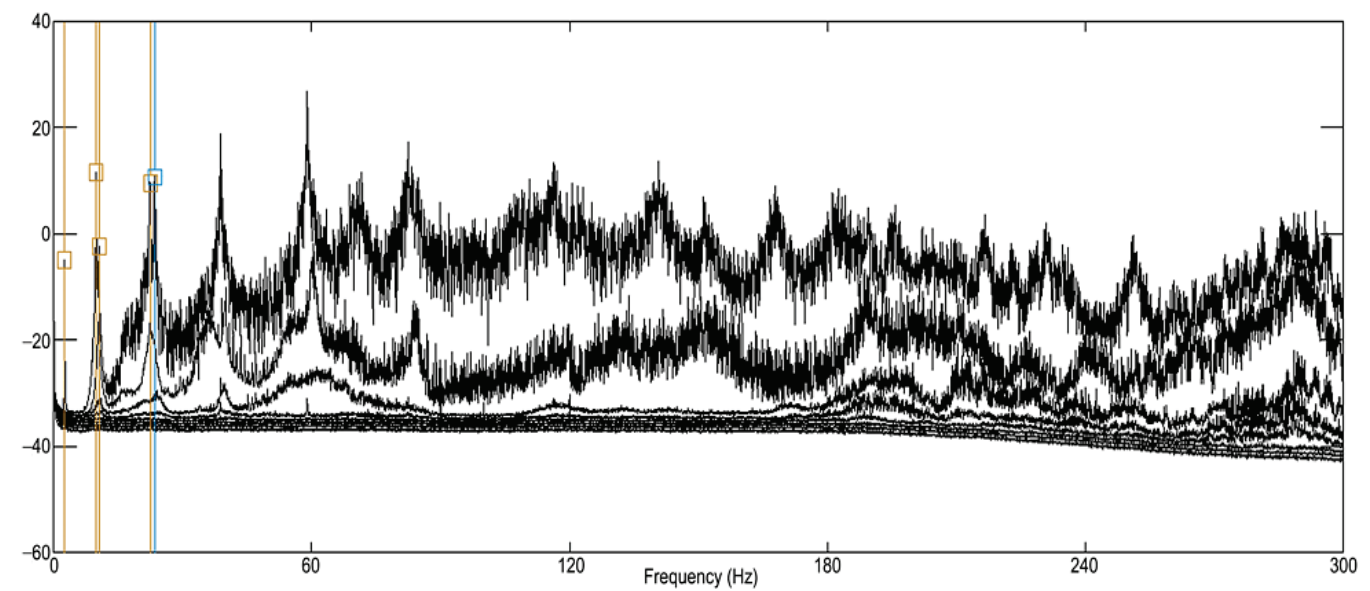

Figure 4 Spectral density matrices with selected five modes using FDD extraction procedure

Table 1 Experimental and numerical modal frequencies for the intact case for both states of the model

\begin{tabular}{|c|c|c|c|c|c|c|c|c|c|}
\hline \multirow{3}{*}{$\begin{array}{l}\dot{0} \\
z \\
0 \\
\dot{0} \\
\Sigma\end{array}$} & \multicolumn{4}{|c|}{ Without Additional Mass } & \multicolumn{4}{|c|}{ With Additional Mass } & \multirow{3}{*}{$\begin{array}{c}\text { Mode shape } \\
\text { Character } \\
/\end{array}$} \\
\hline & \multicolumn{2}{|c|}{ Frequencies } & \multicolumn{2}{|c|}{$\begin{array}{c}\text { Differences in Modal } \\
\text { Frequencies }\end{array}$} & \multicolumn{2}{|c|}{ Frequencies } & \multicolumn{2}{|c|}{$\begin{array}{c}\text { Differences in Modal } \\
\text { Frequencies } \\
\end{array}$} & \\
\hline & $\begin{array}{c}\text { Experimental } \\
/ \mathrm{Hz}\end{array}$ & $\begin{array}{l}\text { Initial FE } \\
\text { Model / Hz }\end{array}$ & $\begin{array}{l}\text { Difference: } \\
\text { Exp-Init. / Hz }\end{array}$ & $\begin{array}{c}\text { Relative } \\
\quad / \%\end{array}$ & $\begin{array}{c}\text { Experimental } \\
/ \mathrm{Hz}\end{array}$ & $\begin{array}{l}\text { Initial FE } \\
\text { Model / Hz }\end{array}$ & $\begin{array}{l}\text { Difference: } \\
\text { Exp-Init. / Hz }\end{array}$ & $\begin{array}{c}\text { Relative } \\
/ \%\end{array}$ & \\
\hline 1 & 2,490 & 2,488 & $+0,002$ & $+0,08$ & 2,050 & 1,930 & $+0,110$ & $+5,59$ & $1^{\text {st }}$ Bending \\
\hline 2 & 9,814 & 9,943 & $-0,129$ & $-1,31$ & 8,090 & 7,720 & $+0,360$ & $+4,56$ & $2^{\text {nd }}$ Bending \\
\hline 3 & 10,550 & 10,835 & $-0,285$ & $-2,70$ & 8,310 & 8,290 & $+0,020$ & $+0,26$ & $1^{\text {st }}$ Torsion \\
\hline 4 & 22,410 & 22,328 & $+0,082$ & $+0,37$ & 18,230 & 17,260 & $+0,970$ & $+5,34$ & $3^{\text {rd }}$ Bending \\
\hline 5 & 23,580 & 23,492 & $+0,088$ & $+0,37$ & 18,640 & 17,870 & $+0,760$ & $+4,08$ & $2^{\text {nd }}$ Torsion \\
\hline
\end{tabular}

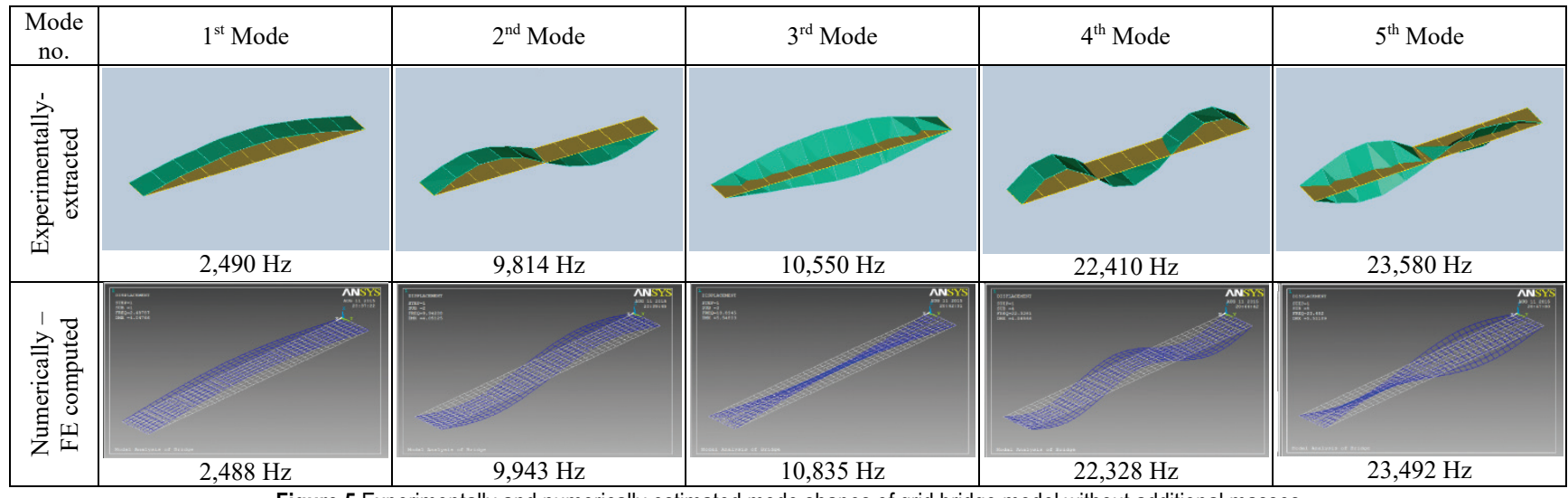

Figure 5 Experimentally and numerically estimated mode shapes of grid bridge model without additional masses

In the state of the model with additional mass, it is simulated in FE structural model using mass element type Mass 21 with mass of 7,925 kg and mass moment of inertia of $0,07925 \mathrm{~kg} \mathrm{~m}^{2}$. Differences between experimentally and numerically estimated frequencies for the first five modes by the initial FE model are listed in Tab. 1. Also, mode shapes correspond to mode shapes for the state without additional mass shown in Fig. 5, with different mode frequency values, as listed in Tab. 1. Differences between modal frequency values are the consequence of the influence of added mass, which is why the corresponding frequency values are reduced.

\section{FE MODEL CALIBRATION BASED ON MODAL PROPERTIES}

There are usually some differences between numerically simulated and experimentally estimated modal properties due to inaccuracy in modelling of the complex real structures [12]. Because of that, the FE model has to 
be calibrated to closely correspond to the actual structure, using the so-called Finite Element Model Updating technique.

\subsection{Calibration of FE Model without Additional Mass}

In this study, the calibration of model without additional mass was implemented using the SA optimization procedure with linear cooling schedule and temperature reduction factor of 0.8 between the two subsequent iterations. The initial temperature during simulation of the cooling process was assumed to be 20 , as an initial parameter.

The SA calibration procedure was based on the comparison of mode frequencies by variation of five parameters. The following parameters: thickness of Rectangular Hollow Section t, t_RHS, of both main longitudinal girders, thickness of T-Section Flange $t$, t_TSF of all longitudinal stiffeners, the width of Rectangular Solid Hidden Beam Sections w, w_RSHBS, the steel material modulus of elasticity E, of all structural elements and the mass density, $\rho$ of all elements were selected for model updating.

The choice of those five parameters was made based on the possible uncertainty of initial parameter values and their high sensitivity of natural frequencies with respect to minor changes of those parameters. The levels of change of those parameters were assumed to be in the range of \pm 7 $\%$, except for the width of parameter $\mathrm{w}_{-}$RSHBS in the range of $\pm 20 \%$, according to their estimated uncertainty.

The measure of difference between experimentally extracted and numerically estimated modal frequencies is represented by the residue $R\left(p_{k}=1,2, \ldots, K\right)$, for the actual values of $K=5$ uncertain parameters, $k=1,2, \ldots, K$ including the first $n=5$ modal frequencies $i=1,2, \ldots, n$. Model calibration is performed by minimization of the residue, in the form of minimization of the objective function, Eq. (1):

$$
O b j_{-} F u n=R\left(p_{k=1,2, \ldots, K}\right)=\sum_{i=1}^{n}\left(\frac{f_{i}^{\text {Tested }}-f_{i}^{\text {Updated }}}{f_{i}^{\text {Tested }}}\right)^{2},
$$

where $f_{i}^{\text {Tested }}$ and $f_{i}^{\text {Updated }}$ are the experimentally and numerically estimated modal frequencies during model updating, respectively.

The calibration process of FE grid bridge model without additional mass is performed by minimizing the proposed objective function Eq. (1), using the SA optimization procedure implemented in MATLAB routine. According to the assumed range of adopted parameters for updating procedure, discretized by four to nine values in the range, the total search space has 17496 possible solutions. The termination criterion is specified by the maximum number of iterations of 500, which represents $2,8 \%$ of the total space. Fig. 6 shows the improved correlation during SA iterations for the grid bridge model without additional mass and the optimum value reached after 51 iterations.

Fig. 7 shows the modification of the chosen five parameters during updating process throughout SA iterations, which results in improvement of the objective function minimum for the grid bridge model without additional mass.

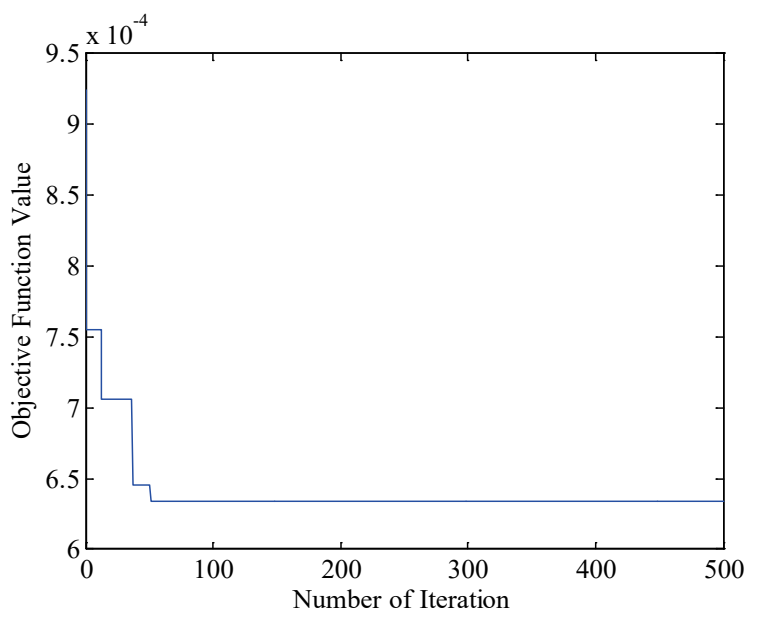

Figure 6 Improvement of FE model correlation according to minimum objective function value by SA optimization procedure

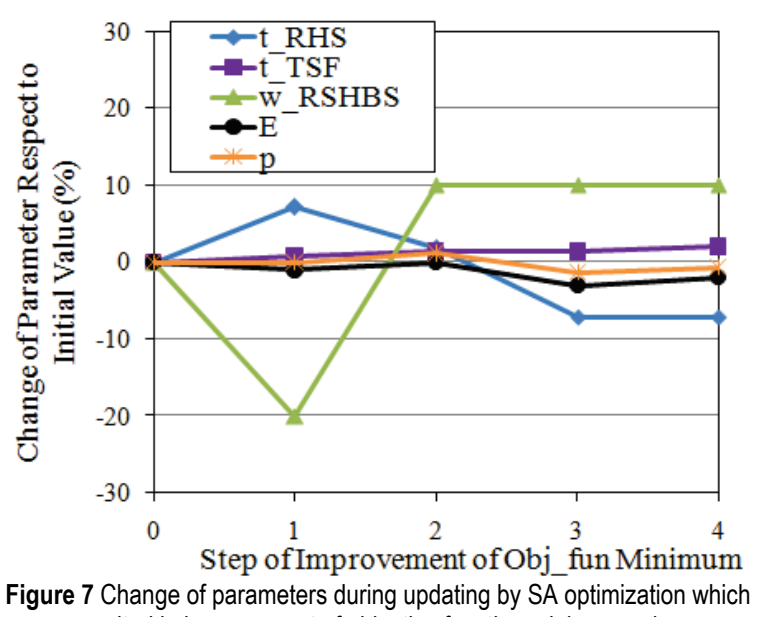
resulted in improvement of objective function minimum value

Calibration results in the optimum values of the varied parameters of, t_RHS_opt $=2,6 \mathrm{~mm}, \mathrm{t}$-TSF_opt $=$ $1,53 \mathrm{~mm}, \mathrm{w} \_\mathrm{RSHBS}$ _opt $=55 \mathrm{~mm}, \mathrm{E} \_$opt $=\overline{1} 196 \mathrm{GPa}$ and $\rho \_$opt $=7,80 \times 10^{3} \mathrm{~kg} / \mathrm{m}^{3}$. The calibrated modal frequencies and corresponding differences with respect to experimental values are listed in Tab. 2 .

The small percentage differences of some initial FE modal frequencies with respect to experimental values, Tab. 2, reflect its high accuracy, improved by adopting the SA updating procedure. The objective function minimum value is improved from the initial value of 0,00093 to 0,00063 for calibrated FE model within four steps, as shown in Fig. 6 and Fig. 7. Consequently, the calibration process with adopted form of the objective function Eq. (1), reduced the high relative differences, thus causing the calibrated FE model to become very close to the real tested model, which is the most important for further damage detection procedure. The herein presented updating procedure proves the efficiency and robustness of the adopted SA optimization for FE model updating according to modal frequencies, where even the initial differences of modal frequencies are small. 
Table 2 Calibrated FE model modal frequencies after applications of SA and TS optimization in comparison with experimentally extracted and initially FE model estimated values

\begin{tabular}{|c|c|c|c|c|c|c|c|c|c|}
\hline & \multirow{3}{*}{$\sum_{\Sigma}^{\frac{0}{0}} \dot{0}$} & \multicolumn{3}{|c|}{ Estimated Modal Frequency } & \multicolumn{5}{|c|}{ Differences in Modal Frequencies } \\
\hline & & \multirow{2}{*}{$\frac{\text { Experimental }}{\text { Exp } / \mathrm{Hz}}$} & \multicolumn{2}{|c|}{ FE Model } & \multicolumn{2}{|c|}{ Error $=$ Exp-Init. } & \multicolumn{3}{|c|}{ Error $=$ Exp-Calib. } \\
\hline & & & Init. / Hz & Calib. / Hz & Diff. / Hz & Relative / \% & Diff. / Hz & Relative / \% & $\begin{array}{l}\text { Relative } \\
\text { Square }\end{array}$ \\
\hline \multirow{6}{*}{ 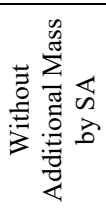 } & 1 & 2,490 & 2,488 & 2,477 & 0,002 & 0,08 & 0,013 & 0,52 & 0,0000273 \\
\hline & 2 & 9,814 & 9,943 & 9,901 & $-0,129$ & $-1,31$ & $-0,087$ & $-0,89$ & 0,0000786 \\
\hline & 3 & 10,550 & 10,835 & 10,740 & $-0,285$ & $-2,70$ & $-0,190$ & $-1,80$ & 0,0003243 \\
\hline & 4 & 22,410 & 22,328 & 22,230 & 0,082 & 0,37 & 0,180 & 0,80 & 0,0000645 \\
\hline & 5 & 23,580 & 23,492 & 23,302 & 0,088 & 0,37 & 0,278 & 1,18 & 0,0001389 \\
\hline & \multicolumn{8}{|c|}{ Residue according to Eq. (1) including the first five modal frequencies } & 0,0006337 \\
\hline \multirow{6}{*}{ 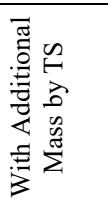 } & 1 & 2,051 & 1,936 & 2,015 & 0,115 & 5,60 & 0,036 & 1,74 & 0,00030 \\
\hline & 2 & 8,093 & 7,724 & 8,039 & 0,369 & 4,56 & 0,054 & 0,66 & 0,00004 \\
\hline & 3 & 8,313 & 8,291 & 8,502 & 0,022 & 0,26 & $-0,189$ & $-2,27$ & 0,00052 \\
\hline & 4 & 18,240 & 17,262 & 17,960 & 0,975 & 5,35 & 0,271 & 1,49 & 0,00022 \\
\hline & 5 & 18,640 & 17,878 & 18,376 & 0,762 & 4,09 & 0,264 & 1,42 & 0,00020 \\
\hline & \multicolumn{8}{|c|}{ Residue according to Eq. (1) including the first five modal frequencies } & 0,00128 \\
\hline
\end{tabular}

\subsection{Calibration of FE Model with Additional Mass}

For the calibration of the model with additional mass, the proposed TS optimization procedure is applied. The search space in the TS procedure is divided into four sets $R$ $=4$, with randomly selected initial point $S_{0}$ inside each set, as well as additional four neighbouring points $N\left(S_{i}\right)_{j}, i=1$, $2, \ldots, 4$, of each set $j=1,2, \ldots, R$, to explore the whole search domain. During the iteration process, the existence of each new generated solution in the so-called Tabu-lists, which memorizes the previously checked solutions, has to be examined. This step is important to avoid the revisiting of checked solutions and preventing the stagnation in local minima in the search space.

In the TS calibration procedure only three parameters are adopted which provide possibility for updating the FE model. These parameters are thickness of Rectangular Hollow Section t, t_RHS, the modulus of elasticity E of all structural elements and the mass density, $\rho$ of all elements with assumed range of change of $\pm 5 \%$ of the three initial parameter values.

The objective function is minimized according to the formula of Eq. (1), with variation of $K=3$ uncertain parameters in the assumed range.

Fig. 8 shows the improvement of objective function minimum value during the calibration using TS optimization. According to the assumed range of adopted parameters for updating procedure, discretized by 29 to 41 values in the range, the total search space includes 47560 possible solutions.

The termination criterion was assumed by the maximum number of possible iterations, limited to 1000 iterations, which represents $2,1 \%$ of the total search space. The optimum value was reached after 880 iterations. The calibrated values of Rectangular Hollow Section t, t_RHS_opt, the modulus of elasticity E_opt and the mass density, $\rho \_$opt are $2,79 \mathrm{~mm}, 210 \mathrm{GPa}$ and $7,46 \times 10^{3} \mathrm{~kg} / \mathrm{m}^{3}$, respectively.

Changes of structural parameters within the permissible range limits are shown in Fig. 9, which resulted in improvement of the objective function minimum approximation. The reduction of differences of modal frequencies for the first five modes is listed in Tab. 2. Even though the applied additional masses increase the complexity of the observed model, the proposed TS optimization procedure during the calibration process exhibits strong performance in convergence of the correlated modes, with significant reduction of modal frequency differences.

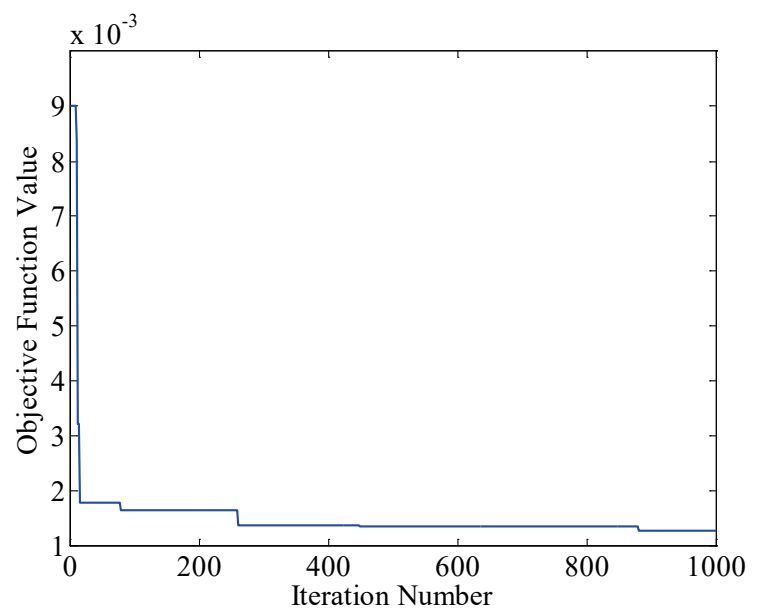

Figure 8 Improvement of FE model correlation according to minimum objective function value by TS optimization procedure

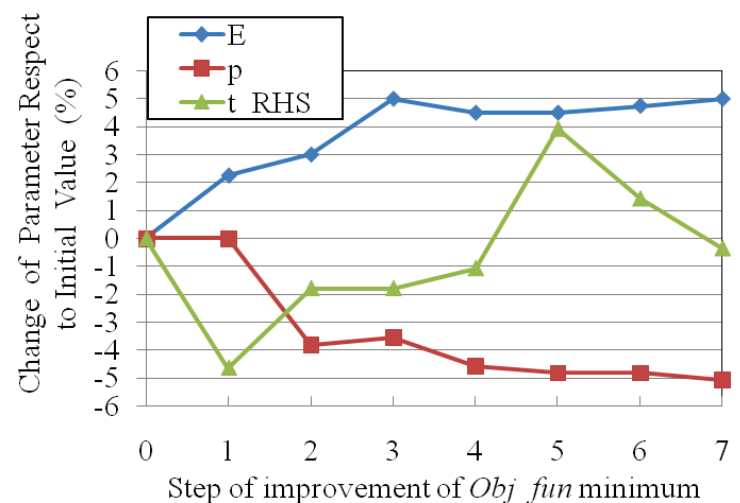

Figure 9 Change of parameters during updating by TS optimization which resulted in improvement of objective function minimum value

The results of numerically extracted natural frequency values before and after updating process with those extracted experimentally, are listed in Tab. 2 for the model with additional mass.

\section{IMPLEMENTED DAMAGE}

In the present study, two cases of different damage scenarios were implemented to investigate the efficiency 
of the proposed procedures, using SA and TS optimization, to detect location and the extent of crack. Both damage scenarios are applied for both cases of the adopted model with and without additional mass and for both damage detection procedures using SA and TS.

\subsection{Adopted Damage Scenarios}

The first implemented damage scenario, DC-1, was represented by damage located at a distance of $2247 \mathrm{~mm}$ $\mathrm{c} / \mathrm{c}$ from support at element no. 54 with damage ratio of 0,4 , crack depth of $20 \mathrm{~mm}$ and crack length of $42 \mathrm{~mm}$, Fig. 1. The damage location in DC-1 was close to the first third of the entire length of longitudinal beam on the left side of the model.
The second implemented damage scenario, DC-2, was represented by the damage located at a distance of 1659 $\mathrm{mm} \mathrm{c} / \mathrm{c}$ from support at element no. 200 with damage ratio of 0,4 , crack depth of $20 \mathrm{~mm}$ and crack length of $42 \mathrm{~mm}$. The second damage location was close to the first quarter of the entire length of the longitudinal beam on the right hand side of the model, Fig. 1. Both damage scenarios were created by a cutter machine from the bottom side of the cross section of the main beams with crack depth of $20 \mathrm{~mm}$ (40\% of the total height of beam cross section which represents ratio of damage 0,4 ) of the total depth of $50 \mathrm{~mm}$, as shown in Fig. 1.

For each damage scenario, the model was first tested without additional mass and then with additional mass.

Also, DC-1 scenario was first examined and repaired, after which the second DC-2 scenario was implemented.

Table 3 Experimentally extracted modal frequencies for intact and implemented damage cases of the both model states

\begin{tabular}{|c|c|c|c|c|c|c|c|c|c|c|}
\hline \multirow{3}{*}{$\begin{array}{c}\text { Mode } \\
\text { No. }\end{array}$} & \multicolumn{5}{|c|}{ Without Additional Mass } & \multicolumn{5}{|c|}{ With Additional Mass } \\
\hline & \multirow{2}{*}{$\begin{array}{c}\begin{array}{c}\text { Intact } \\
\text { Case }\end{array} \\
\text { Freq. / Hz }\end{array}$} & \multicolumn{2}{|c|}{ DC-1 } & \multicolumn{2}{|c|}{ DC-2 } & \multirow{2}{*}{$\begin{array}{c}\text { Intact Case } \\
\text { Freq. / Hz }\end{array}$} & \multicolumn{2}{|c|}{ DC-1 } & \multicolumn{2}{|c|}{ DC-2 } \\
\hline & & $\begin{array}{c}\text { Freq. / } \\
\mathrm{Hz}\end{array}$ & $\begin{array}{c}\text { Reduction / } \\
\%\end{array}$ & Freq. / Hz & $\begin{array}{c}\text { Reduction / } \\
\%\end{array}$ & & Freq. / Hz & $\begin{array}{c}\text { Reduction / } \\
\%\end{array}$ & Freq. / Hz & $\begin{array}{c}\text { Reduction / } \\
\%\end{array}$ \\
\hline 1 & 2,490 & 2,454 & $-1,45$ & 2,490 & 0,00 & 2,050 & 2,050 & 0,00 & 2,010 & 0,02 \\
\hline 2 & 9,814 & 9,705 & $-1,11$ & 9,631 & $-1,86$ & 8,090 & 7,940 & 1,80 & 7,910 & 2,26 \\
\hline 3 & 10,550 & 10,360 & $-1,80$ & 10,360 & $-1,80$ & 8,310 & 8,240 & 0,87 & 8,200 & 1,32 \\
\hline 4 & 22,410 & 22,230 & $-0,80$ & 22,120 & $-1,29$ & 18,230 & 18,090 & 0,80 & 18,050 & 0,98 \\
\hline 5 & 23,580 & 23,400 & $-0,76$ & 23,400 & $-0,76$ & 18,640 & 18,420 & 1,18 & 18,530 & 0,59 \\
\hline
\end{tabular}

The ambient vibration test and estimation procedure for the intact state of the structural model was repeated after repairing the damage created in DC- 1 . In this way, the same modal properties were ensured as in the initial intact case.

\subsection{Experimental Analysis of Damaged Model Based on Ambient Vibration Measurements}

The extracted modal frequencies for the observed damage cases, for the states of model without and with additional mass for the first five modes, are listed in Tab. 3. The percentage of reduction with respect to the corresponding frequencies in the intact case is also shown for both states of model.

In the case of DC-1, different frequency values are extracted for all five modes, while, for the damage case DC-2, different modal frequencies are extracted just for the second to fifth modes. Besides minor differences in frequencies in the range of $2 \%$, there are also certain differences in mode shapes, which could be useful in damage detection process.

\section{DAMAGE DETECTION BY THE PROPOSED PROCEDURES BASED ON HEURISTIC OPTIMIZATION METHODS.}

For damage detection of experimentally studied damage cases, DC-1 and DC-2, two proposed procedures based on SA and TS heuristic optimization methods were applied as routines developed under MATLAB environment. They include numerical simulation of damage parameters (i.e. damaged element number and ratio of damage) of calibrated FE model, and the modal analysis of updated FE model was performed by ANSYS FE package. During the proposed optimization procedures, the FE model is updated and modified by the change of model stiffness (by selection of damage parameters at the current stage), [4], and use for computation value of objective function. The SA optimization method was applied to the observed damaged model without additional mass, while the TS optimization method was applied to the model with additional mass. The parameters representing the damage are damaged element number $N_{\mathrm{D}}$ and ratio of damage $R_{\mathrm{D}}$.

\subsection{Damage Detection Procedure by SA Optimization Method for the Model without Additional Mass}

For damage detection procedure, SA optimization procedure was implemented using its parameters, the linear cooling schedule, temperature reduction factor of 0.8 and assumed initial temperature of 20 . In the neighbourhood of the current configuration, in each SA iteration, six neighbouring solutions are designed to distribute checking points in the searching space. Also, the proposed modified objective function, [7], for damage detection of both adopted damage cases, with weighting factor values is given in Eq. (2):

$$
\begin{aligned}
& f u n_{S A}^{D}=W_{\mathrm{F}} \times \sum_{i=1}^{n}\left(f_{i}^{E}-f_{i}^{N}\right)^{2}+ \\
& +W_{\mathrm{D} 1 \times \sum_{i=1}^{n}}^{n}\left[\sqrt{\sum_{j=1}^{m}\left(\Phi_{i j}^{E}-\Phi_{i j}^{N}\right)^{2}}\right]
\end{aligned}
$$

The symbols $f_{i}^{E}$ and $f_{i}^{N}$ represent natural frequencies extracted from experimental investigation of the structure and numerical computation, respectively, while $n$ represents the number of included modes. Symbols 
$\Phi_{i j}^{E}$ and $\Phi_{i j}^{N}$ represent the normalized mode shape vector components in vertical and horizontal directions at measurement points, estimated based on the experimentally and numerically computed data, respectively. The $m$ is the total number of measuring component points (transducers), located at particular nodes of the FE structural model. The factors $W_{\mathrm{F}}$ and $W_{\mathrm{D} 1}$ are the so-called weighting factors of two parts of the objective function which are adopted 10 and 1 , respectively.

In this study, the ratio of damage was considered in the range of $R_{\mathrm{D}}=[0,01,0,99]$, as a discrete variable with the accuracy (step) of 0,01 . According to the created FE model, the damaged element identification number is in the range of the total number of elements $N_{\mathrm{D}}=[1,320]$, taking into the account just finite elements of the main longitudinal beams of the structural model, Fig. 1. The search space is defined by the number of all possible solutions of 31680 , which is very high and offers the possibility for accurate determination of damage ratio.

Due to the width of the search space, the maximum number of iterations was limited to 1000 , i.e. $3,2 \%$ of the total possible solutions (points) number in search space. It defines one of the termination criteria, while the second termination criteria is temperature when the system becomes frozen, i.e. $T<1$, according to the SA principle. The procedure, with the proposed objective function form, includes the first five modes with their frequencies and two transverse displacement components of the mode shape vectors.

Improvement of the objective function during damage detection (via SA optimization process) for the studied scenario-1 of damaged model without additional mass is shown in Fig. 10 (a). Distribution of checked solutions during the process in the search space is shown in Fig. 10 (b). A very quick improvement of the optimal solution approximation, Fig. 10 (a), and convergence during SA optimization can be observed, without achieving the prescribed maximum number of iterations. The optimum solution was reached after 839 iterations (with percentage $2,6 \%$ of whole search space). It closely corresponds to the actual parameters of damage produced in DC-1. The procedure detected position of damage on element $N_{\mathrm{D}}^{*}=47$ with damage ratio of element $R_{\mathrm{D}}^{*}=0,52$, Fig. 10 (b) (i.e. damage crack $=26 \mathrm{~mm}$ ), while the actual damage parameters are $N_{\mathrm{D}}=54$ and $R_{\mathrm{D}}=0,40$ (i.e. damage crack $=20 \mathrm{~mm}$ ).
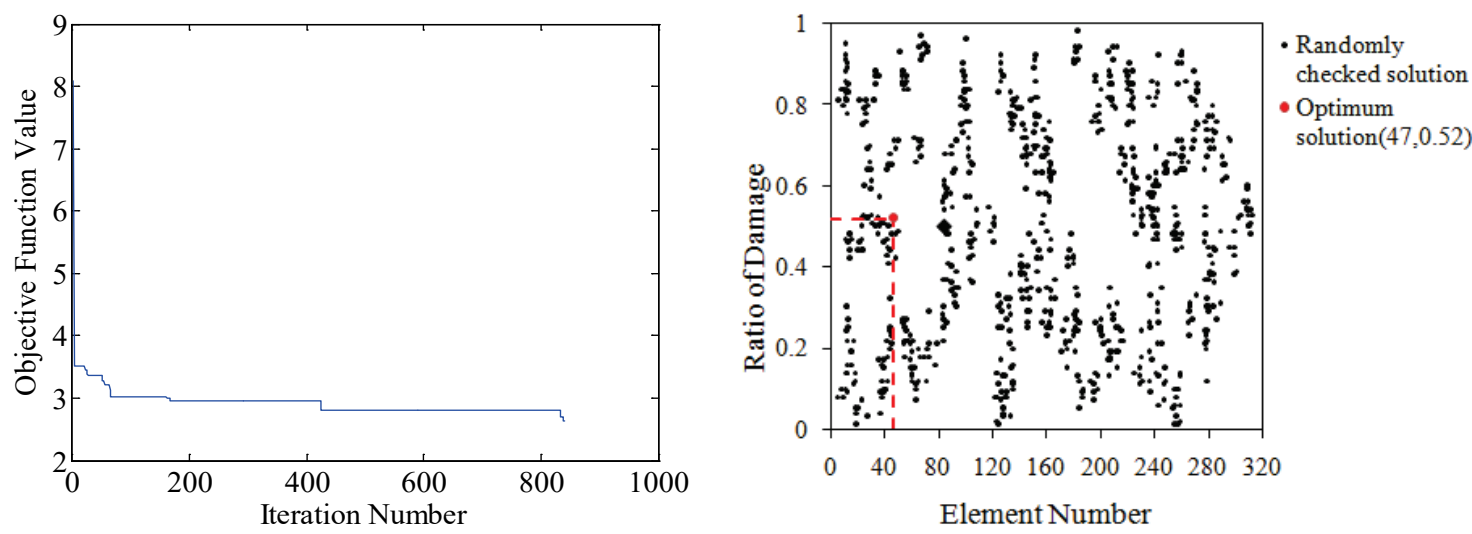

(a)

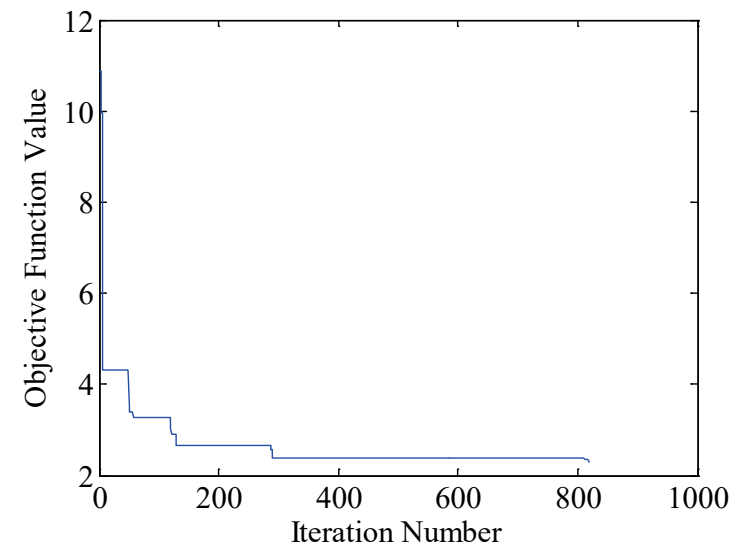

(c)

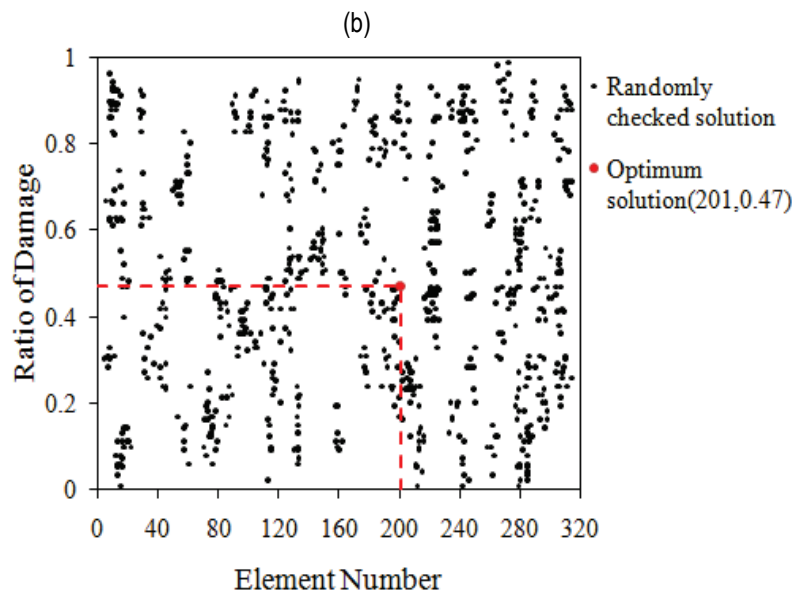

(d)

Figure 10 Improvement of the objective function global minimum estimation during the proposed SA based damage detection procedure for the studied DC-1 (a) with distribution of checked points in the search space (b) for the model without additional mass and for the studied DC-2 (c) distribution of checked points in the search space (d) for the model without additional mass

Generated solutions in the search space, Fig. 10 (b) show that the process covers the whole search space to avoid local minimums and explore the global optimum solution. A small percentage of the total number of solutions was checked, just a $2,6 \%$ of the total number of possible solutions.
The proposed damage detection procedure based on the SA optimization, for the scenario-2, DC-2, exhibits quick convergence, Fig. 10 (c). The optimal solution was reached after 819 iterations (the number of checked solutions), which represents $2,6 \%$ of the total possible solutions. In this case, the procedure detected very close 
position of damage on element $N_{\mathrm{D}}^{*}=201$ with the damage ratio of $R_{\mathrm{D}}^{*}=0,47$ (i.e. damage crack $=23,5 \mathrm{~mm}$ ), Fig. $10(\mathrm{~d})$. The optimum solution is very close to the actual damage scenario DC-2, represented by damage parameters $N_{\mathrm{D}}=200$ and $R_{\mathrm{D}}=0,40$ (i.e. damage crack depth of $20 \mathrm{~mm}$ ). As in the previous case, the search solutions are also randomly distributed throughout the whole search space, Fig. 10 (d).

\subsection{Damage Detection Procedure by TS Optimization Method for the Model with Additional Mass}

For the proposed TS optimization procedure for damage detection, the objective function consists of three parts which takes into the account mode shape absolute value differences of normalized vectors and the third part represents the orthogonality between mode shapes using the MAC parameter, [13], are defined by Eq. (3).

The adopted values of weighting factors $W_{\mathrm{F}}, W_{\mathrm{D} 2}$ and $W_{\mathrm{M}}$ are $1,0,1$ and 1 , respectively.

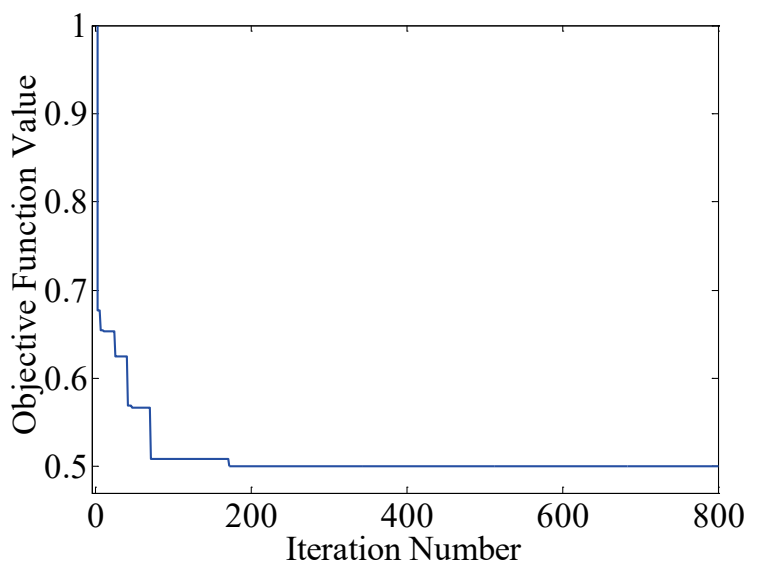

(a)

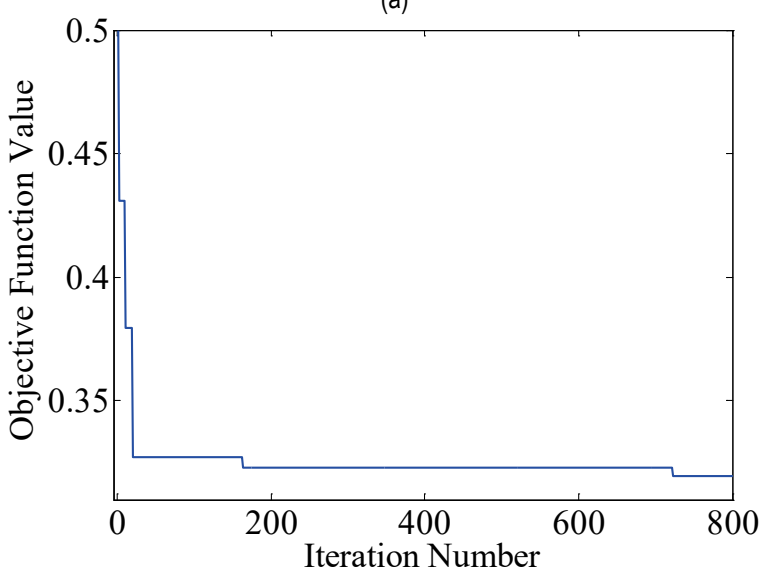

(c)

Figure 11 Improvement of the objective function global minimum estimation during the proposed TS based damage detection procedure for the studied DC-1 (a) with distribution of checked point in the search space (b), for the case of model with additional mass and for the studied DC-2 (c) with distribution of checked point in the search space (d), for the model with additional mass

Quick improvement of the objective function optimal value approximation, during the damage detection by the proposed TS optimization procedure for the studied Scenario-1 of damaged model with additional mass, is shown in Fig. 11 (a). The optimum solution is achieved after only 172 iterations (with percentage $1,1 \%$ of whole search space). The distribution of checked solutions during the optimization process in the search space is presented in

$$
\begin{aligned}
& f u n_{\mathrm{TS}}^{\mathrm{D}}=W_{\mathrm{F}} \sum_{i=1}^{n}\left(f_{i}^{E}-f_{i}^{N}\right)+W_{\mathrm{D} 2} \sum_{i=1}^{n}\left[\sqrt{\sum_{j=1}^{m}\left(\left|\Phi_{i j}^{E}\right|-\left|\Phi_{i j}^{N}\right|\right)^{2}}\right]+ \\
& +W_{\mathrm{M}} \sum_{i=1}^{n}\left[1-\left(\frac{\left(\left|\Phi_{i j}^{N}\right|^{\mathrm{T}} \cdot\left|\Phi_{i j}^{E}\right|\right)^{2}}{\left(\left(\left|\Phi_{i j}^{N}\right|^{\mathrm{T}} \cdot\left|\Phi_{i j}^{N}\right|\right) \times\left(\left|\Phi_{i j}^{E}\right|^{\mathrm{T}} \cdot\left|\Phi_{i j}^{E}\right|\right)\right)}\right)\right],
\end{aligned}
$$

In this study, the ratio of damage was assumed to be in the range of $R_{\mathrm{D}}=[0,02,0,94]$ with increment of 0,02 . The damaged element was considered to be in the range of $N_{\mathrm{D}}$ $=[1,320]$, elements of the main longitudinal beams. The number of total possible solutions in the search space, according to the considered variables and accuracy, was 15 040. The search space was divided into 20 sets $R=20$ while the neighbouring solutions were nine solutions $N\left(S_{i}\right)_{j}, i=1,2, \ldots, 9$. The maximum number of iterations, (maximum number of checked solutions) was limited to 800 as termination criterion, which represents $5,3 \%$ of all possible solutions in the adopted TS damage detection procedure.

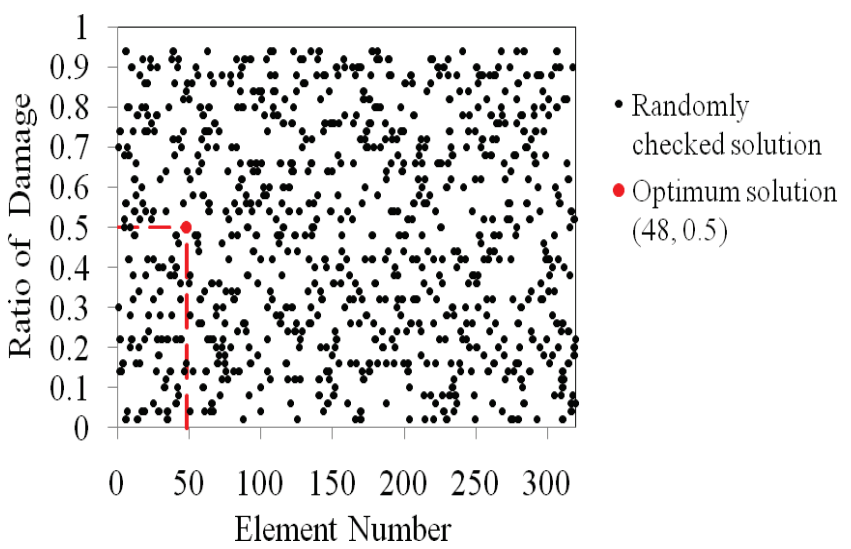

(b)

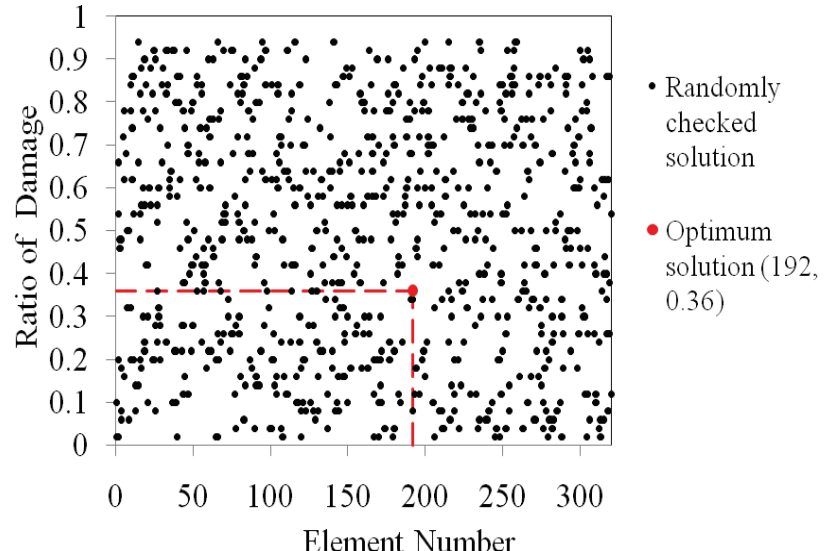

(d) Number

(d)
Fig. 11 (b), which indicates that the whole search space is covered by the proposed TS optimization procedure. This fact reflects the efficiency of the proposed optimization TS procedure for application to this type of problems. The proposed damage detection procedure based on TS optimization method explored the position of damage on element $N_{\mathrm{D}}^{*}=48$ with damage ratio of $R_{\mathrm{D}}^{*}=0,50$ (i.e. 
damage crack $=25 \mathrm{~mm})$, Fig. $11(\mathrm{~b})$. The actual parameters of damage, for the DC- 1 case, are $N_{\mathrm{D}}=54$ and $R_{\mathrm{D}}=0,40$ (i.e. damage crack $=20 \mathrm{~mm}$ ), and the explored values are close to the actual damage parameters.

The optimum solution for the studied case DC-2 of damage was reached after 723 iterations (number of checked solutions before the optimum was reached), which represents $4,8 \%$ of the whole search space, Fig. 11 (c), with the close values of damage parameters with respect to the actual. The proposed procedure, based on TS optimization, explored the location of damage at the damaged element $N_{\mathrm{D}}^{*}=192$, which is quite close to the actual location at damaged element $N_{\mathrm{D}}=200$.

The explored ratio of damage of $R_{\mathrm{D}}^{*}=0,36$ (i.e. damage crack $=18 \mathrm{~mm})$, is very close to the actual ratio of damage $R_{\mathrm{D}}=0,40$ (i.e. damage crack $=20 \mathrm{~mm}$ ), in the DC2 damage scenario, Fig. 11 (d).

Distribution of the checked solutions during damage detection by the proposed TS procedure reflects the ability of covering the whole search domain with a minimum number of iterations, as shown in Fig. 11 (d).

\section{CONCLUSION}

Ambient vibration measurements were carried out for two states of the model, with and without additional mass, in order to cover wider frequency range which exists in the case of real structures. In both states, accurate modal frequencies with numerically estimated mode shapes were extracted based on the high-quality measurements with a small number of transducers. Extraction process is provided via application of FDD method, which is the state-of-the-art for such application.

Accurate modal frequencies, with respect to experimental results, were estimated by initial FE modeling, with differences in the range of 5,5\%. According to high importance of compatibility between FE estimated and experimentally extracted modal properties for damage detection, the improvement of the initial model was conducted via calibration process, even with minor initial differences.

The calibration of initial FE models, conducted using the proposed SA and TS optimization based procedures for the state without and with additional mass respectively, resulted in the excellent agreement of modal properties, with differences in the range of $2,5 \%$ with respect to the precise and experimentally estimated modal frequencies.

The proposed forms of the objective function with adopted weighting factors exhibit high sensitivity in changes in modal properties for the adopted damage scenarios in both model states, and recognize the small changes, less than $2,3 \%$ with respect to the intact case, in experimentally extracted modal frequencies. For both damage scenarios, the adopted objective functions used in SA and TS optimization methods, by the global minimum accurately reflect the actual damage parameters. The efficiency of the proposed procedures is proven by damage application at two different locations on the structural model, with different influence on the change of the modal properties of the first five modes considered.

The proposed damage detection and calibration procedures based on the SA optimization technique, exhibit high accuracy in detecting damage location and extent with a quick convergence in the large search space. Also, based on the adopted accuracy, the damage parameters, damaged element and ratio of damage, were closely explored for both implemented cases. The proposed TS optimization procedure, in both calibration and damage detection processes, has a quick convergence and high accuracy for detecting the optimal solutions, which were achieved according to the ability to explore a large search space within the limited number of iterations. The proposed procedures with excellent agreement regarding the conducted experiments prove the reliability for structural health monitoring application of complex real structures.

\section{Acknowledgments}

The present work is part of investigation within the research project TR-36048: Research on condition assessment and improvement methods of civil engineering structures in view of their serviceability, load-bearing capacity, cost effectiveness and maintenance, financed by the Ministry of Education, Science and Technological Development of the Republic of Serbia. The authors would like to express their gratitude to the Ministry of Education, Science and Technological Development of the Republic of Serbia within the project entitled "World in Serbia" and the Ministry of Higher Education and Scientific Research of the Republic of Iraq.

\section{REFERENCES}

[1] Kang, F., Junjie, L., \& Sheng, L. (2013). Combined data with particle swarm optimization detection. Journal of Mathematical Problem in Engineering, article ID 416941, 10 pages. https://doi.org/10.1155/2013/416941

[2] He, R. \& Hwang, S. (2007). Identifying Damage in Spherical Laminate Shells by Using a Hybrid Real-Parameter Genetic Algorithm. Journal of Composite Structures, 80(1), 32-41. https://doi.org/10.1016/j.compstruct.2006.02.035

[3] Guggenberger, J. (2005). In search of simulating reality: validation and updating of FE models for structural analysis. International Congress on FEM Technology with ANSYS $C F X \&$ ICEM CFD Conference, 1-10.

[4] Mišković, Z., Al-Wazni, S., Alalikhan, A., \& Salatić, R. (2014). Methods of Damage Detection of Structures Based on Changes of Dynamic Characteristics. Construction Calendar of the Union of Engineers and Technicians of Serbia, 47, 163-191.

[5] Hui, L., Kegui, X., \& Quanquan, Q. (2011) Study of Structural Damage Detection with Multi-Objective Function Genetic Algorithms. Procedia Engineering of SREE of Int. conference on Engineering Modeling and Simulation / Chain, 80-86. https://doi.org/10.1016/j.proeng.2011.05.014

[6] He, R. \& Hwang, S. (2006). Damage Detection by an Adaptive Real-Parameter Simulated Annealing Genetic Algorithm. Journal of Computers and Structures, 84(31-32), 2231-2243. https://doi.org/10.1016/j.compstruc.2006.08.031

[7] Au, F., Cheng, Y., Tham L., \& Bai, Z. (2003). Structural Damage Detection Based on a Micro-Genetic Algorithm Using Incomplete and Noisy Modal Test Data. Journal of Sound and Vibration, 259(5), 1081-1094. https://doi.org/10.1006/jsvi.2002.5116

[8] Michel, G. \& Jean, P. (2014). Chapter 6: Tabu Search, in Search Methodologies / Introductory Tutorials in 
Optimization and Decision Support Techniques / $2^{\text {nd }}$ edition, Ed. Edmund K. Burke and Graham Kendall, Springer. https://doi.org/10.1007/0-387-28356-0_6

[9] Kirkpatrick, S., Gelatt, C., \& Vecchi, M. (1983). Optimization by Simulated Annealing. Journal of Science (Jstor), 220(4598), 671-680. https://doi.org/10.1126/science.220.4598.671

[10] Al-Wazni, S., Alalikhan, A., Mišković, Z., \& Salatić, R. (2014). Numerical Test of Damage Detection Using Simulated Annealing Method. Proceedings of the $14^{\text {th }}$ Int. conference of ASES - Association of Structural Engineering of Serbia, ed. M. Lazović, B. Stevanović / Serbia, 513-520.

[11] Alalikhan, A., Al-Wazni, S., Mišković, Z., \& Salatić, R. (2014). Numerical Test of Damage Detection Using Tabu Search Method. Proceedings of the $14^{\text {th }}$ Int. conference of ASES - Association of Structural Engineering of Serbia, ed. M. Lazović, B. Stevanović / Serbia, 513-520.

[12] Sehgal, S. \& Kumar, H. (2015). Structural Dynamic Model Updating Techniques: a State of the Art Review. Journal of Arch Computat Methods Eng. https://doi.org/10.1007/sl 1831-015-9150-3

[13] Ruotolo, R. \& Suarce, C. (1997). Damage Assessment of Multiple Cracked Beams: Numerical Results and Experimental Validation. Journal of Sound and Vibration, 206(4), 567-588. https://doi.org/10.1006/jsvi.1997.1109

\section{Contact information:}

Zoran MIŠKOVIĆ, PhD

Structural Engineering Department,

Faculty of Civil Engineering, University of Belgrade

Bulevar kralja Aleksandra 73,

11000 Belgrade, Republic of Serbia

E-mail: mzoran@imk.grf.bg.ac.rs

\section{Saad AL-WAZNI, MSc}

Department of Civil Engineering,

Faculty of Engineering, University of Kufa, Iraq

$\mathrm{PhD}$ student at the Structural Engineering Department of the Faculty of Civil

Engineering, University of Belgrade

E-mail: al_wazni@yahoo.com

Ahmed ALALIKHAN, MSc

Department of Civil Engineering,

Faculty of Engineering, University of Kufa, Iraq

$\mathrm{PhD}$ student at the Structural Engineering Department of the Faculty of Civil

Engineering, University of Belgrade

E-mail: ahmed.alikan@gmail.com 